\title{
Enfoque práctico para medir anemia dilucional
}

\author{
Practical approach to measure dilutional anemia
}

\section{Señor Editor:}

En la práctica clínica habitual se administra grandes volúmenes de fluidos intravenosos a los pacientes como parte del manejo de trauma, cirugía o reposición de volumen, práctica que está asociada a una condición denominada hemodilución iatrogénica (HI). La HI se define como el aumento del volumen plasmático causado por cualquier intervención médica, usualmente por la administración de cristaloides (1).

La importancia de la $\mathrm{HI}$ radica en que predispone al paciente a anemia dilucional, ya que el aumento del volumen plasmático sin un incremento proporcional de la masa de glóbulos rojos produce una reducción paradójica del transporte de oxígeno, incremento del riesgo de sangrado por coagulopatía dilucional y reducción de la temperatura corporal $(2,3)$. Asimismo, se sabe que la respuesta fisiológica adaptativa a la HI se produce 15 a 20 minutos después de la administración, por el efecto de distribución; bajo esta premisa es recomendado no administrar algún tipo de tratamiento $(4,5)$.

Para la evitar un escenario en el que se administre tratamientos a un paciente con anemia dilucional deberíamos considerar la siguiente pregunta: ¿Existe alguna manera de saber si un paciente tiene anemia dilucional por HI?

La respuesta es afirmativa, ya que en un estudio de reciente publicación (6), se propuso un enfoque para medir la anemia dilucional en 4 simples pasos.
Se inicia con el cálculo del volumen sanguíneo total pre infusión, una forma simple es la estimación de $65 \mathrm{ml} / \mathrm{kg}$ de peso para mujeres y $75 \mathrm{ml} / \mathrm{kg}$ para varones. Segundo, calcular la cantidad de hemoglobina corporal total (HCT) multiplicando la concentración de hemoglobina en g/dl por el volumen sanguíneo total inicial. El tercer paso es calcular el volumen sanguíneo final post-infusión, para esto se debe considerar que por cada litro de solución salina normal aproximadamente un cuarto del volumen permanece en el cuerpo, mientras que para la solución de dextrosa al 5\% (D5W) permanece únicamente una duodécima parte. Por último, se calcula la concentración esperada de hemoglobina post-infusión dividiendo el valor de HCT entre el volumen sanguíneo post-infusión.

Aplicar este enfoque en las situaciones necesarias podrá ayudar a discernir la caída en la concentración de hemoglobina por administración de fluidos o por una etiología diferente y evitar tratamientos iatrogénicos.

Finalmente, se recomienda que en la práctica clínica habitual se consideren los conceptos de HI por administración de fluidos intravenosos y anemia dilucional, y sean cuantificados de formas simples, ya que no existen guías de práctica clínica para el manejo de tales escenarios. Los hospitales públicos y privados deben considerar este problema en sus directrices de práctica clínica.

Harold André Guerrero-Martínez ${ }^{1, a}$, Fresia Cicibel Casas-Bueno ${ }^{1, a}$, Alonzo Rojas-Barahona 1,a, Joel Gonzales-Mendieta ${ }^{1, a}$, Felix Cuti-Simon ${ }^{1, a}$

\footnotetext{
1 Facultad de Medicina Alberto Hurtado, Universidad Peruana Cayetano Heredia. Lima, Perú.

a Estudiante.
} 


\section{Correspondencia:}

Harold André Guerrero Martínez

Urb. El Oasis D5- Código postal 15109

San Martín de Porres, Perú

Correo electrónico: harold.guerrero.m@upch.pe

Celular: 51961537062

\section{REFERENCIAS BIBLIOGRÁFICAS}

1. Bowdler AJ, Renshaw FG. Dilutional Anemia. In: Bowdler AJ, editor. The Complete Spleen: Structure, Function, and Clinical Disorders. Totowa, NJ: Humana Press; 2002. p. 123-35.

2. Perel A. Iatrogenic hemodilution: a possible cause for avoidable blood transfusions? Crit Care Lond Engl. 2017 25; 21(1):291.
3. Cronin JA, Oetgen ME, Gordish-Dressman H, Martin BD, Khan N, Pestieau SR. Association between perioperative surgical home implementation and transfusion patterns in adolescents with idiopathic scoliosis undergoing spinal fusion. Pediatr Anesth. 2019; 29(6):611-9.

4. Smith A. A comprehensive guide to the management of anaemia. Prescriber. 2015;26(8):11-20.

5. Hahn RG, Lyons G. The half-life of infusion fluids: An educational review. Eur J Anaesthesiol. 2016; 33(7):475-82.

6. Hale AJ, Ricotta DN, Herzig SJ, William JH, Freed JA. A quantitative approach to dilutional anemia. J Hematol. 2019; 8(2):86-87.

Recibido: 14/04/2020

Aceptado: 05/07/2020 